\title{
Équilibres de phases en chromatographie micellaire. Corrélation LSER de la constante d'équilibre micelle-eau
}

\author{
S. Guermouche et M.H. Guermouche \\ Faculté de Chimie, USTHB, BP. 32 El-Alia, Bab-Ezzouar, Alger, Algérie
}

\begin{abstract}
Summary : Micellar liquid chromatography allows to determine micelle-water equilibrum constant $K_{M W}$. Capacity factors of fify solutes of various polarities are measured using cationic and anionic micellar mobilephase and a $\mathrm{C}_{18}$ silica column. The solvatochromic parameters of the solutes are used to establish a convenient LSER (linear solvation equation relationship) correlations for the capacity factors. Using the Armstrong model, the micelle-water equilibrum constant $K_{M W}$ of the solutes used are determined. d'Armstrong. The LSER equations found for $K_{\text {HWW }}$ give a a straights with a correlation coefficient higher than 0.95 . For the two used surfactants, The LSER coefficients found allow to conclude that the size of the solute is the most influencial parameter on micelle-water equilibrum constant $K_{M W}$ followed by the solute basicity.
\end{abstract}

\section{INTRODUCTION}

Contrairement à la chromatographie en phase liquide classique (CPL) dans laquelle le soluté se distribue entre deux phases, en chromatographie micellaire (CLM), le soluté se partage entre les trois phases en présence : la phase stationnaire, la micelle et la phase mobile extramicellaire. La CLM permet de déterminer les constantes d'équilibre correspondantes et plus particulièrement la constantes d'équilibre micelle-eau $K_{M W}$.

Dans ce travail, en utilisant des phases mobiles micellaires anioniques (à base de dodécyle sulfate de sodium), et cationiques ( à base de bromure de cétyltriméthyl ammonium) et une phase stationnaire en silice greffée octadécyle, les facteurs de capacité d'une cinquantaine de solutés de différentes polarités seront déterminés. Par ailleurs, Les paramètres solvatochromiques pris de la littérature [1] seront utilisés pour établir des corrélations LSER (linear solvation equation relationship) du facteur de capacité des solutés utilisés. En utilisant le modèle d'Armstrong [2,3], Les constantes d'équilibre micelle-eau $K_{M W}$ de tous les solutés seront déterminées

\subsection{Partie théorique}

La CLM introduit 3 équilibres comme le montre la figure 1. Dans la phase mobile, deux phases en équilibre ( constante d'équilibre $K_{M W}$ apparaissent, la phase aqueuse contenant des monomères de surfactant, la phase micellaire formée d'agrégats de molécules de surfactant. Les deux phases précédemment décrites sont en équilibre avec la phase stationnaire; les constantes respectives sont $K_{S W}$ et $K_{S M}$. La rétention d'un soluté dans le système chromatographique est régie dans ce cas par les trois constantes; dans la chromatographie à polarité de phases inversée classique, un seul équilibre existe. 


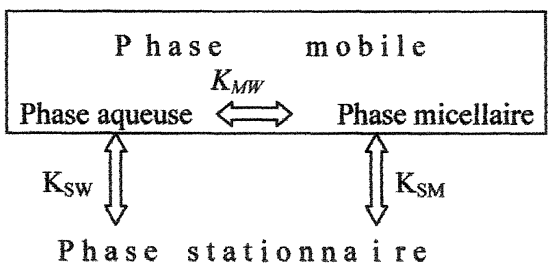

Figure 1. Schéma de principe de la chromatographie liquide micellaire

\subsubsection{Modèle d'Armstrong [2,3]}

Le modèle théorique d'Armstrong permet d'étudier la rétention d'un soluté en CLM. Le facteur de capacité $k^{\prime}$ d'un soluté est donné par

$$
1 / k^{\prime}=\left[\gamma^{\prime} \phi\right]\left[\left(K_{M W}-1\right) / K_{S W}\right] \cdot C m+1 /\left(\phi . K_{S W}\right)
$$

$k^{\prime}:$ facteur de capacité du soluté

$\gamma$ : Volume molaire partiel du surfactant

$\mathrm{Cm}$ : Concentration micellaire

$\phi:$ rapport des volumes de phases $=V s / V o$

$1 / k$ ' est une droite en fonction de $\mathrm{Cm}$

Le rapport pente/ordonnée à l'origine permet d'avoir $K_{M W}$, constante d'équilibre soluté-micelle par monomère de surfactant. Pour avoir la constante équivalente par micelle, il faut multiplier $K_{M W}$ par le nombre d'agrégats dans une micelle.

\subsubsection{Paramétrisation LSER [1,4]}

Kamlet, Abraham et Carr [1] ont proposé une multiparamétrisation reliant un paramètre de rétention aux propriétés du soluté suivant :

$$
\mathrm{SP}=\mathrm{SPo}+\mathrm{mV} \mathrm{V}_{1} / 100+\mathrm{rR}+\mathrm{s} \pi^{*}+\mathrm{b} \beta+\mathrm{a} \alpha
$$

SP est la propriété du soluté ; $\ln k^{\prime}$ ou $\ln K_{M W} \mathrm{~V}_{1}$ est le volume intrinsèque du soluté

$\mathrm{R}$ est l'excès de réfraction molaire du soluté

$\pi^{*}$ mesure la polarity/polarisability du soluté, $\beta$ est sa basicité, $\alpha$ son acidité.

$\mathrm{SPo}, \mathrm{m}, \mathrm{r}, \mathrm{s}, \mathrm{b}$, a sont les paramètres correspondants.

\subsection{Partie expérimentale}

L'appareil HPLC utilisé a les caractéristiques suivantes :Waters Chromatograph ALC/GPC 244 avec pompe $6000 \mathrm{~A}$, injecteur Rheodyne 7125 et détecteur UV Jasco UV 975 opérant à $254 \mathrm{~nm}$. Les séparations sont effectuées sur une colonne Spherisorb C18-5 ( $0.39 \times 15 \mathrm{~cm}$ ) précédée d'une colonne de garde $(4 \times 0.4 \mathrm{~cm})$ remplie de Corasil II C18.

Plusieurs phases mobiles contenant différentes concentrations de surfactants et du propanol-2 comme modificateur organique ont été utilisées.

Les solutés employés appartiennent à différentes familles à polarités variables. 


\subsection{Résultats et discussion}

\subsubsection{Modèle d'Armstrong}

En utilisant l'équation (1), 1/k' est porté en fonction de la concentration micellaire $\mathrm{Cm}$ du surfactant.

Les droites obtenues permettent de constater que le modèle d'Armstrong décrit convenablement la rétention en CLM. Le tableau 1 donne quelques valeurs de $K_{M W}$ obtenues sur le SDS et CTAB.

\begin{tabular}{|l|c|c|}
\hline Soluté & SDS & CTAB \\
\hline Benzene & 287 & 94 \\
Benzonitrile & 224 & 137 \\
Nitrobenzene & 283 & 190 \\
Phenol & 176 & 147 \\
Chlorobenzene & 457 & 362 \\
Acenaphthylene & 744 & 1058 \\
Acenaphthene & 1674 & 417 \\
Pyrene & 1125 & 1130 \\
Triphenylene & 1550 & 1173 \\
benzo $(a)$ pyrene & 3720 & 1342 \\
dibenzo $(a, h)$ anthracene & 4898 & 1540 \\
Fluorene & 2356 & 609 \\
Phenanthrene & 1488 & 968 \\
Anthracene & 1702 & 621 \\
Pentacene & 274 & 3975 \\
Dibenzo $(a, c)$ anthracene & 5642 & 1711 \\
& & \\
\hline
\end{tabular}

Tableau 1. Quelques valeurs de $K_{M W}$ obtenues sur le SDS et CTAB.

Si nous considérons la série des hydrocarbures polyaromatiques, les micelles anioniques donnent de plus grandes valeurs de $K_{M W}$.que les micelles cationiques à base de CTAB.. Ceci veut dire que la micelle anionique attire davantage les hydrocarbures polyaromatiques que la micelle cationique. De plus, avec la micelle à base de SDS, l'effet géométrique est important puisque l'hydrocarbure le plus droit, le pentacène, a la constante la plus faible tandis que l'hydrocarbure le moins droit, le dibenzo $(a, c)$ anthracene possède le plus grand $K_{M W}$. De ce fait, une molécule droite trouve plus de facilité à s'insérer dans la micelle anionique.

\subsubsection{Paramétrisation LSER}

La figure 2 montre la paramétrisation LSER des constantes $K_{M W}$ dans le cas de la CLM cationique ou anionique en utilisant des hydrocarbures polyaromatiques, des solutés polaires ou la totalité des solutés.

Pour les surfactants cationique ou anionique, une correlation relativement convenable est obtenue (coefficient de correlation compris entre 0.96 et 0.97 ), ceci est du à la difficulté de transfert du soluté. Dans les corrélations obtenues, il semble que les facteurs les plus importants sont $\beta$ et $\mathrm{V}$. Pour le SDS, l'effet de $b \beta$ est négatif. La basicité du soluté décroit $K_{M W}$ entrainant une diminution de la liaison du soluté à la micelle anionique. Ces solutés se lient plus facilement à une micelle cationique. D'un autre côté, les solutés les plus volumineux interagissent plus avec une micelle anionique. 


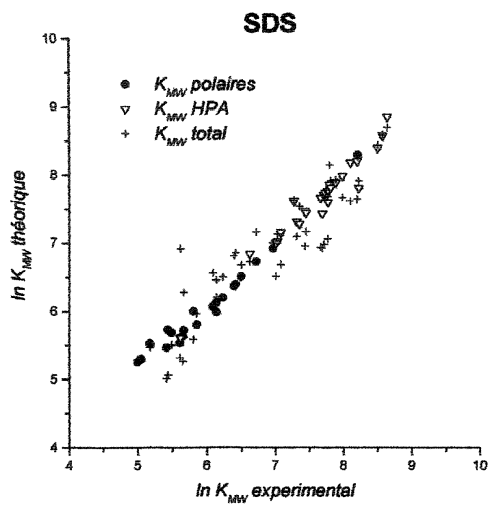

a)

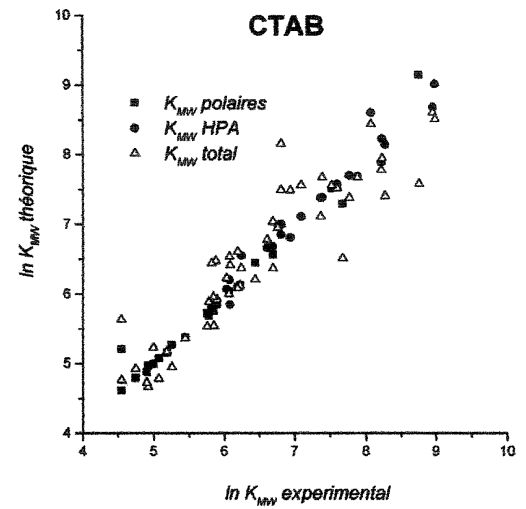

b)

Figure 2. Paramétrisation LSER des constantes $K_{M W}$ dans le cas du SDS (a) et le CTAB (b).

\subsection{Conclusion}

Les résultats indiquent que le modèle d'Armstrong décrit bien la rétention de solutés de différentes polarités en chromatographie micellaire anionique ou cationique. Il apparait que l'introduction d'un hydrocarbures polyaromatiques dans une micelle anionique est plus facile.La corrélation LSER de $K_{M W}$ est satisfaisante pour les micelles cationiques et anioniques.Dans cette corrélation, les facteurs prépondérants sont la basicité (effet négatif) et le volume (effet positif)

\section{Références}

1. M.J. Kamlet, M.H. Abraham, P.W. Carr P.W., R.M. Doherty , R.W. Taft, J.Chem.Soc. Perkin Trans. II,2087 (1988).

2. D.W. Armstrong, S.J. Henry, J. Liq. Chromatogr., 3,657 (1980).

3. M. Arunyanat, L.J. Cline Love, Anal. Chem., 57,2837 (1985).

4. M.A. Garcia, M.F. Vitha, M.I. Marina, J.Liq.Chrom.\& Rel.Technol., 23,873 (2000). 